\title{
Análise das prescrições de medicamentos na atenção primária à saúde de um município Centro-potiguar
}

\author{
Analysis of drug prescriptions in primary health care in a city in the Centro-potiguar \\ Análisis de prescripción de medicamentos en atención primaria de salud en una ciudad del \\ Centro-potiguar
}

\author{
Christyan de Azevedo Jota1 (1), Almária Mariz Batista² (1) \\ ¿Universidade Federal do Rio Grande do Norte, Faculdade de Farmácia - Natal (RN), Brasil. \\ 2Universidade Federal do Rio Grande do Norte, Escola Multicampi de Ciências Médicas -Caicó (RN), Brasil.
}

\section{Resumo}

Introdução: A não conformidade de prescrições de medicamentos com normativas que regulamentam esse processo é uma realidade do processo de cuidado em saúde.

Objetivo: Este estudo teve como objetivo analisar prescrições médicas e odontológicas do sistema primário de saúde do município de Angicos, Rio Grande do Norte de acordo com a legislação em vigor. Métodos: Desenvolveu-se estudo transversal de caráter descritivo-exploratório. Constituíram objeto de análise segundas vias de prescrições e receituário de controle especial elaborados por todos os médicos e odontólogos lotados na atenção primária desse município, no período de janeiro a junho de 2018. Verificaram-se itens como presença de nome do paciente, idade, sexo, via de administração, dosagem/concentração, duração do tratamento, nomenclatura do medicamento, carimbo e assinatura do prescritor. Resultados: Analisaram-se 3.725 prescrições, em que $566(15,19 \%)$ estavam não conformes com a legislação, sendo mais problemáticos os itens identificação do usuário $(68,02 \%)$, data da prescrição $(34,10 \%)$, nomenclatura do medicamento por denominação comum brasileira/internacional $(18,02 \%)$, duração do tratamento $(13,96 \%)$ e posologia (10,60\%). Conclusões: Esses resultados apontam para a necessidade de que estratégias de educação permanente e fiscalização qunto ao cumprimento da legislação sejam desenvolvidas de forma mais assídua.

Palavras-chave: Assistência farmacêutica; Prescrições de medicamentos; Atenção primária à saúde.
Como citar: Jota CA, Batista AM. Análise das prescrições de medicamentos na atenção primária à saúde de um município Centro-potiguar. Ver Bras Med Fam Comunidade. 2022;17(44):2432. https://doi.org/10.5712/ rbmfc17(44)2432

\author{
Autor correspondente: \\ Almária Mariz Batista \\ E-mail: almariamariz@yahoo.com.br \\ Fonte de financiamento: \\ nao se aplica. \\ Parecer CEP: \\ 83029518.0.0000.5292. \\ Procedência: \\ não encomendado. \\ Avaliação por pares: \\ externa. \\ Recebido em: 09/03/2020. \\ Aprovado em: 02/10/2021. \\ Editor Associado: \\ Thiago Dias Sarti
}




\begin{abstract}
Introduction: The non-compliance of drug prescriptions with regulations that control this process is a reality of the health care process. Objectives: Thus, this study aimed to analyze medical and dental prescriptions of the primary health system in the city of Angicos-Rio Grande do Norte, in accordance with the current law. Methods: Accordingly, a cross-sectional descriptive-exploratory study was conducted. Second copies of prescriptions and special control prescriptions prepared by all physicians and dentists working in primary health care in this municipality, from January to June 2018, were analyzed. Information included the patient's name, age and sex. route of administration, dosage/concentration, duration of treatment, drug nomenclature, and stamp and signature of the prescriber. Results: A total of 3,725 prescriptions were analyzed, in which $566(15.19 \%)$ were not in compliance with the law, where most problematic were user identification (68.02\%), date of prescription (34.1\%). drug nomenclature $(\mathrm{DCB} / \mathrm{DCl})(18.02 \%)$, duration of treatment $(13.96 \%)$ and dosage $(10.6 \%)$. Conclusions: These results point to the need for permanent education and inspection strategies regarding compliance with the law to be developed more assiduously.
\end{abstract}

Keywords: Pharmaceutical services; Drug prescriptions; Primary health care.

\title{
Resumen
}

Introducción: El incumplimiento de la prescripción de medicamentos con la normativa que regula este proceso es una realidad del proceso asistencial. Objetivo: Así, este estudio tuvo como objetivo analizar las prescripciones médicas y odontológicas del sistema primario de salud de la ciudad de Angicos-RN, de acuerdo con la legislación vigente. Métodos: Por tanto, se desarrolló un estudio descriptivo-exploratorio transversal. Se analizaron segundas copias de prescripciones y prescripciones especiales de control elaboradas por todos los médicos y odontólogos que laboran en la atención primaria de este municipio, de enero a junio de 2018. Se verificaron ítems como la presencia del nombre del paciente, edad, sexo, administración, dosis/concentración, duración del tratamiento, nomenclatura del fármaco, sello y firma del prescriptor. Resultados: Se analizaron un total de 3.725 prescripciones, de las cuales $566(15,19 \%)$ no cumplieron con la legislación, siendo más problemáticos los ítems identificación de usuario $(68,02 \%)$, fecha de prescripción $(34,1 \%)$, nomenclatura de medicamentos. DCB / DCl $(18,02 \%)$, duración del tratamiento $(13,96 \%)$ y posología (10,6\%). Conclusiones: Estos resultados apuntan a la necesidad de que se desarrollen con mayor asiduidad estrategias de educación continua y de inspección sobre el cumplimiento de la legislación.

Palabras clave: Servicios farmacéuticos; Prescripciones de medicamentos; Atención primaria de salud.

\section{INTRODUÇÃO}

A Organização Mundial de Saúde (OMS) instituiu, em 2017, o Terceiro Desafio Global de Segurança do Paciente, Medicação Sem Danos, cuja meta é reduzir erros de medicação em $50 \%$ até 2023. ${ }^{1}$ Nessa conjuntura, estudos no âmbito da atenção primária constataram incidentes de segurança relacionados à medicação como os mais prevalentes ${ }^{2-4}$ e a etapa de prescrição de medicamentos como a mais susceptível a erros de medicação. ${ }^{5-7}$

Além disso, estima-se que mais de $50 \%$ dos medicamentos são prescritos, dispensados e utilizados de forma não racional e cerca de $40 \%$ dos usuários atendidos na atenção primária não necessita de medicamento, contudo $80 \%$ destes saem das consultas com prescrição medicamentosa. ${ }^{8-10}$

A prescrição constitui documento para comunicação escrita entre profissionais da saúde e entre estes e o usuário, ${ }^{11}$ portanto o problema inicia-se com a comunicação inadequada entre o profissional da farmácia que dispensa o medicamento e o prescritor que o prescreve. Nesse processo, a falha na comunicação constitui a principal causa de erros, ${ }^{2-4}$ e isso impacta de forma direta a saúde do paciente, já que este é o usuário dos medicamentos prescritos.

No Brasil, visando minimizar erros de medicação, aprovaram-se a Lei n 5.991/73, a Portaria $n^{\circ} 344 / 98$, a Lei n ${ }^{\circ} 9.787 / 99$ e a Resolução $n^{\circ} 20 / 11,{ }^{12-15}$ para manter profissionais de saúde atualizados e capacitados a prestarem adequado serviço multiprofissional aos usuários do sistema de saúde.

Apesar da legislação, a fiscalização quanto ao seu cumprimento ocorre de forma esporádica, o que gera margem para que erros de prescrição continuem acontecendo. Entretanto, é responsabilidade ética 
do prescritor redigir a prescrição de forma adequada, com esclarecimentos e disponibilidade perante possíveis efeitos indesejáveis. ${ }^{16}$

Ante esse contexto, o objetivo deste estudo é analisar as prescrições médicas e odontológicas da atenção primária à saúde do município de Angicos (RN), a fim de se avaliar sua conformidade com a Lei $n^{\circ}$ 5.991/73, a Lei n 9.787/99, a Portaria n 344/98 e a Resolução n 20/11.

\section{MÉTODOS}

Constitui estudo transversal, de caráter descritivo-exploratório, com abordagem quantitativa, no âmbito da atenção primária à saúde de um município localizado na região central do Rio Grande do Norte. Trata-se de Angicos, cuja população, conforme último censo (2010), abrangia 11.549 habitantes, sendo estimado 11.695 habitantes para $2021 .^{17}$

No contexto da atenção primária, à época de coleta e análise dos dados, esse município dispunha de cinco unidades básicas de saúde/estratégias de saúde da família (UBS/ESF), sendo quatro na zona urbana e uma na zona rural, além de uma central de abastecimento farmacêutico (CAF), a qual também centralizava o processo de dispensação de medicamentos nesse nível de cuidado via sua farmácia central.

Nesse sentido, avaliaram-se todas as segundas vias das prescrições e todo receituário de controle especial elaborados no período de janeiro a junho de 2018 por médicos e odontólogos lotados nas cinco UBS/ESF, arquivados na farmácia central. A coleta de dados foi realizada por um graduando em farmácia mediante treinamento prévio.

Consideraram-se os itens exigidos na Lei n $5.991 / 73$, Lei n $5.787 / 99$, Portaria n $344 / 98$ e Resolução $n^{\circ} 20 / 11^{12-15}$ para identificar a conformidade das prescrições averiguadas. Dessa forma, a conformidade das prescrições foi avaliada mediante os itens presença de nome, idade e sexo do paciente, carimbo/ assinatura e categoria profissional do prescritor, data da prescrição, via de administração, concentração, duração do tratamento, nomenclatura dos medicamentos prescritos conforme denominação comum brasileira/internacional ( $\mathrm{DCB} / \mathrm{DCl})$, conformidade entre categorização do medicamento e respectivo tipo de prescrição, conformidade entre quantidade de medicamento e respectivo tipo de prescrição, conformidade entre quantidade de unidade posológica e respectivo tipo de prescrição, identificação do receptor, nome/carimbo/assinatura do farmacêutico e data da dispensação.

Prescrições contendo mais de um medicamento foram consideradas não conformes para cada um dos itens via de administração, concentração e duração do tratamento, caso não houvesse sido informado. Também foram consideradas não conformes as que não apresentavam, concomitantemente, carimbo e assinatura do prescritor.

Adicionalmente, verificou-se a conformidade das prescrições com a lista de medicamentos essenciais, a qual se baseou na Relação Municipal de Medicamentos Essenciais (REMUME 2017), ${ }^{18}$ versão mais atual à época de coleta e análise de dados.

Não houve critério para avaliar legibilidade dado o curto período de tempo para coleta e análise dos dados, consequentemente, houve limitação para montar equipe para verificar parâmetros que tornam uma prescrição ilegível, pois esse item pode ser considerado subjetivo quando analisado por um único pesquisador.

Os dados estatísticos foram tratados com auxílio do software Microsoft Office Excel versão 365, e as informações obtidas em porcentagem foram tabuladas para melhor compreensão.

Realizou-se coleta dos dados mediante aprovação pelo Comitê de Ética do Hospital Universitário Onofre Lopes (CEP/HUOL), sob número de protocolo 83029518.0.0000.5292. 


\section{RESULTADOS}

Ao todo, analisaram-se 3.725 prescrições, das quais, 566 (15,2\%) estavam não conformes.

A maioria das prescrições analisadas foi elaborada por médicos (92,0\%), e em $0,1 \%$ não foi possível constatar o tipo de prescritor por não constar seu carimbo e assinatura. Entre as prescrições não conformes, 92,8\% foram elaboradas por médicos (Tabela 1).

Tabela 1. Distribuição das prescrições quanto ao tipo de prescritor.

\begin{tabular}{lcccc}
\hline Prescritor & Total de prescrições & $\mathbf{( \% )}$ & $\mathbf{N}^{\circ}$ de prescrições não conformes & (\%) \\
\hline Médico & 3.427 & 92,0 & 525 & 92,8 \\
Odontólogo & 294 & 7,9 & 37 & 6,5 \\
Não identificado & 4 & 0,1 & 4 & 0,7 \\
TOTAL & 3.725 & 100 & 566 & 100 \\
\hline
\end{tabular}

Dessa forma, quanto às razões de total de prescrições e total de prescrições não conformes entre médicos e odontólogos, constatou-se, respectivamente, 11,7 ( 12) e 14,2 ( 14), ou seja, médicos elaboraram 12 vezes mais prescrições e 14 vezes mais prescrições não conformes. Isso posto, considerando-se que essas razões se equiparam, depreende-se que o nível de não conformidade entre prescrições elaboradas por médicos e odontólogos também se equiparam.

Entre as prescrições avaliadas, não se identificou nenhuma regida pela Portaria $n^{\circ} 344 / 98$ elaborada por odontólogo. Todo o receituário elaborado por esse profissional continha medicamentos isentos de prescrição e antimicrobianos.

A avaliação dos itens das prescrições conforme legislação em vigor ${ }^{12-15}$ estão apresentados na Tabela 2.

Tabela 2. Distribuição das prescrições não conformes quanto aos itens avaliados.

\begin{tabular}{lcc}
\hline Item & Prescrições não conformes & (\%) \\
\hline Lei no 5.991/73 & $385(41,3)$ & 68,02 \\
Identificação do usuário & 193 & 34,10 \\
Data da prescrição & 79 & 13,96 \\
Duração de tratamento & 60 & 10,60 \\
Posologia & 19 & 3,40 \\
Via de administração & 13 & 2,30 \\
Concentração & 13 & 2,30 \\
Carimbo e assinatura do prescritor & 10 & 1,80 \\
Informações que permitam localizar prescritor & 9 & 1,60 \\
Nome do medicamento & & 18,02 \\
Lei no 9.787/99 & 102 & 0 \\
Nomenclatura do medicamento por DCB/DCl & & 0 \\
RDC no 20/11 & 0 & 0 \\
Idade do usuário & 0 & \\
Sexo do usuário & 0 & \\
Carimbo e assinatura do farmacêutico & & \\
\hline
\end{tabular}


Tabela 2. Continuação.

\begin{tabular}{lcc}
\hline Item & Prescrições não conformes & (\%) \\
\hline Portaria no 344/98 & 21 & 3,70 \\
Identificação da pessoa que recebeu o medicamento & 6 & 1,06 \\
Tipo de prescrição e quantidade de unidade posológica & 1 & 0,20 \\
Carimbo e assinatura do farmacêutico & 1 & 0,20 \\
Escrituração registrada em livros específicos & 0 & 0 \\
Tipo de prescrição e quantidade de medicamento & 0 & 0 \\
Tipo de prescrição e categorização do medicamento & & \\
\hline
\end{tabular}

DCB/DCl: denominação comum brasileira/denominação comum internacional.

Ao todo, identificaram-se 933 não conformidades nas prescrições, resultando em média de 4 não conformidades/prescrição. Os itens mais prevalentes quanto à não conformidade foram identificação do usuário $(68,02 \%)$, data da prescrição $(34,10 \%)$, nomenclatura do medicamento por $\mathrm{DCB} / \mathrm{DCl}(18,02 \%)$, duração do tratamento $(13,96 \%)$ e posologia $(10,60 \%)$, sendo os dois primeiros mais prevalentes no receituário de controle especial.

Quanto às prescrições sujeitas à Portaria n 344/98, constataram-se 442 prescrições não conformes com essa norma. Desse total, 3,70\% não apresentavam identificação da pessoa que recebeu o medicamento, 1,06\% não estava em conformidade entre tipo de prescrição e quantidade de unidade posológica e em $0,20 \%$ não constava carimbo e assinatura do farmacêutico atestando dispensação, consequentemente, não constava escrituração registrada em livro específico, somente via HÓRUS.

Todos os medicamentos presentes nas prescrições analisadas constavam na REMUME $2017 .{ }^{18}$

\section{DISCUSSÃO}

Houve prevalência de prescrições elaboradas por médicos (92\%), realidade constatada por diversos autores como em UBS de Teresina (PI) $(60,8 \%),{ }^{19}$ em UBS de Porto Alegre (RS) $(99,7 \%)^{20}$ e em farmácia básica de Pontal do Araguaia (MT) $(98,8 \%) .{ }^{21}$

Isso pode ser explicado pelo fato de que usuários procuram mais atendimento médico que dos demais profissionais da atenção básica. Segundo último levantamento realizado via DATASUS, registraram-se no Rio Grande do Norte $4.186(69,8 \%)$ visitas a médicos e $1.814(30,2 \%)$ para os demais profissionais do núcleo de saúde. ${ }^{22}$ Entretanto, houve equiparação entre as razões de total de prescrições ${ }^{12}$ e total de prescrições não conformes ${ }^{14}$ entre médicos e odontólogos, o que sugere que a quantidade de prescrições elaboradas não interferiu na quantidade de prescrições não conformes.

Um dado preocupante foi a presença de quatro $(0,1 \%)$ prescrições aviadas sem qualquer tipo de identificação de quem prescreveu, o que aponta fragilidades no processo de dispensação. Esse dado é essencial para contato com o prescritor em caso de dúvidas, tanto por parte do usuário quanto do farmacêutico. ${ }^{23}$

A maior prevalência de não conformidade foi relacionada ao item ausência de identificação do usuário $(68,02 \%)$. O que torna o dado alarmante é o fato de que a prescrição só pode ser aviada mediante presença obrigatória desse item, ${ }^{12}$ logo, isso alerta para ocorrência de dispensação/distribuição mesmo com essa não conformidade. Além disso, a presença desse item evita destinação do medicamento a terceiros, levando a seu uso não racional, e oportuniza diálogo com o usuário. 
A ausência de identificação do usuário em Angicos foi 358 vezes superior ao constatado em UBS de Ouro Preto (MG) $(0,19 \%),{ }^{24} 28,3$ vezes maior que o identificado em centro de saúde de Lajeado (RS) $(2,40 \%)^{25}$ e 6,8 vezes a mais que o verificado em UBS de Teresina (10,00\%). ${ }^{19}$ Essa discrepância pode ser explicada pelas diferentes metodologias aplicadas a cada estudo, apesar do objetivo de se analisar o mesmo elemento.

Para o item não conforme data da prescrição (34,1\%), esse também superou resultados de estudos desenvolvidos em farmácia básica de Pontal do Araguaia $(0,8 \%),{ }^{21}$ em UBS de Ouro Preto $(0,19 \%),{ }^{24}$ na atenção primária de Caicó (RN) $\left(0,0 \%\right.$ e 1,6\%, respectivamente), ${ }^{26,27}$ em UBS de ljuí (RS) $(0,8 \%)^{28}$ e em uma instituição de utilidade pública do Vale do Aço (MG) $(8,6 \%){ }^{29}$

Angicos utiliza o sistema HÓRUS, por meio do qual assistentes de farmácia e farmacêuticos conseguem, pelo histórico de dispensação, verificar a última vez que o medicamento de uso contínuo foi entregue a determinado usuário. Isso reduz o risco desse ser distribuído/dispensado antes da data prevista no sistema, contudo não anula a não conformidade com a legislação nem resolve o problema, pois há medicamentos de uso não contínuo, além da necessidade de se averiguar a data de validade da prescrição, a fim de reduzir a tendência à automedicação.

Quanto ao item não conforme nomenclatura do medicamento por $\mathrm{DCB} / \mathrm{DCl}(18,02 \%)$, no âmbito do Sistema Único de Saúde (SUS) é obrigatória a prescrição de medicamentos considerando esse item. ${ }^{12}$ Além disso, a OMS preconiza meta de $100 \%$ de medicamentos prescritos por nome genérico ${ }^{30}$ a fim de facilitar educação, informação, acesso e promoção de seu uso racional. ${ }^{31}$

Esses dados corroboram revisões sistemáticas de avaliação de indicadores de prescrição de medicamentos na atenção primária, nas quais se constatou que todos os estudos analisados continham medicamentos não prescritos por $\mathrm{DCB} / \mathrm{DCl} .{ }^{32,33}$ Além disso, superam resultados constatados na atenção primária de Caicó $(14,6 \% \text { e 5,7\%, respectivamente })^{26,27}$ e em farmácia comunitária de Sobral (CE) $(4,82 \%) .{ }^{34}$

Quanto aos itens não conformes duração do tratamento $(13,96 \%)$ e posologia $(10,60 \%)$, sua ausência ou apresentação de forma incompleta pode comprometer a efetividade e a segurança da terapia farmacológica, pois pode levar o usuário a utilizar o medicamento de forma equivocada.

Estudos de avaliação do nível de conhecimento de usuários sobre prescrições de medicamentos na atenção primária de Piripiri $(\mathrm{PI})^{35}$ e Ribeirão Preto $(\mathrm{SP})^{36}$ constataram fragilidades quanto ao nome do medicamento/duração do tratamento e à posologia, respectivamente, revelando os riscos a que os usuários estão expostos pelo entendimento ineficaz de suas prescrições.

Esses dados se equiparam aos constatados em UBS de Teresina (16,10\%), ${ }^{19}$ em UBS de Ouro Preto $(17,42 \%)^{24}$ e em UBS de ljuí $(16,10 \%),{ }^{28}$ no caso, para duração do tratamento. Em contrapartida, dados de duração de tratamento são superados pelos constatados na atenção primária de Caicó $(97,1 \%$ e 40,8\%, respectivamente), ${ }^{26,27}$ o que pode ser explicado pelo fato desses dois estudos se restringirem a grupos terapêuticos específicos (psicofármacos e antimicrobianos, respectivamente).

A não conformidade das prescrições com a Portaria $n^{\circ} 344 / 98$ também é realidade constatada em farmácia comunitária de Sobral, ${ }^{34}$ onde $0,3 \%$ do receituário analisado não apresentava informações do usuário, e na farmácia básica de Pontal do Araguaia, ${ }^{21}$ onde se constatou que em $6,0 \%$ das prescrições analisadas não constava identificação do receptor, em 0,4\% havia divergência entre quantidade de unidade posológica e respectivo tipo de prescrição e em $100 \%$ não constava registro do responsável pela dispensação.

Em Angicos, 442 (79,5\%) prescrições não conformes era receituário de controle especial, o que se torna preocupante, pois, conforme legislação vigente, o não cumprimento de suas exigências constituirá infração sanitária, ficando o infrator sujeito a penalidades. ${ }^{14} \mathrm{O}$ conhecimento do prescritor sobre o que 
é prescrito e o reconhecimento do valor da presença do farmacêutico, elo entre usuários e equipe de saúde ${ }^{37}$ são fatores essenciais para evitar não conformidades em prescrições.

A não conformidade das prescrições analisadas neste estudo com a legislação em vigor, além de superar resultados constatados em outros estudos de análise de prescrições de medicamentos na atenção primária, ${ }^{19,21,24-29,34}$ contraria recomendações internacionais para prescrição de medicamentos efetiva e segura..$^{30,31,38}$

\title{
CONCLUSÃO
}

Aotodo, 566 (15,2\%) prescrições analisadas estavam não conformes comalegislação em questão, sendo mais problemáticos os itens identificação do usuário $(68,02 \%)$, data da prescrição $(34,10 \%)$, nomenclatura do medicamento por DCB/DCl $(18,02 \%)$, duração do tratamento $(13,96 \%)$ e posologia $(10,60 \%)$.

Os resultados deste estudo apontam para a necessidade de que educação permanente e fiscalização do cumprimento da legislação em questão por prescritores de medicamentos sejam desenvolvidas de forma mais assídua, além da necessidade de reorientação do processo de formação profissional, mediante práticas mais seguras de elaboração da prescrição de medicamentos.

Ante o exposto, gestores e profissionais de saúde dispõem de subsídios para que estratégias efetivas de melhoria da qualidade da prescrição de medicamentos, priorizando seus aspectos mais problemáticos, sejam aplicadas no contexto da atenção primária do município em estudo, a fim de contribuir para efetividade da terapia farmacológica e segurança do paciente.

\section{CONTRIBUIÇÕES DOS AUTORES}

CAJ: Conceituação, Análise Formal, Investigação, Metodologia, Administração do Projeto, Visualização, Escrita - Primeira Redação. AMB contribuiu com Conceituação, Curadoria de Dados, Análise Formal, Metodologia, Administração do Projeto, Validação, Visualização, Escrita - Revisão e Edição.

\section{CONFLITOS DE INTERESSES}

\author{
Nada a declarar.
}

\section{REFERÊNCIAS}

1. World Health Organization. Medication without harm: global patient safety challenge on medication safety. Geneva: World Health Organization; 2017.

2. Marchon SG, Mendes Junior WV. Segurança do paciente na atenção primária à saúde: revisão sistemática. Cad Saúde Pública 2014;30(9):1815-35. https://doi.org/10.1590/0102-311X00114113

3. Panesar SS, Silva D, Carson-Stevens A, Cresswell KM, Salvilla SA, Slight SP, et al. How safe is primary care? A systematic review. BMJ Qual Saf 2016;25(7):544-53. https://doi.org/10.1136/bmjqs-2015-004178

4. Medeiros SG, Virgílio LA, Santos VEP. Segurança do paciente na atenção primária: uma scoping review. Rev APS 2019;22(2):423-39. https://doi.org/10.34019/1809-8363.2019.v22.16743

5. Olaniyan JO, Ghaleb M, Dhillon S, Robinson P. Safety of medication use in primary care. Int J Pharm Pract 2015;23(1):3-20. https://doi.org/10.1111/ijpp.12120

6. Bates DW, Cullen DJ, Laird N, Petersen LA, Pequeno SD, Servi D, et al. Incidence of adverse drug events and potential adverse drug events. JAMA 1995;274(1):29-34. https://doi.org/10.1001/jama.1995.03530010043033

7. Montserrat-Capella D, Suárez M, Ortiz L, Mira JJ, Duarte HG, Reveiz L. Frequency of ambulatory care adverse events in Latin American countries: the AMBEAS/PAHO cohort study. Int J Qual Health Care 2015;27(1):52-9. https://doi.org/10.1093/ intqhc/mzu100 
8. World Health Organization. Medicines: rational use of medicines. Geneva: World Health Organization; 2010.

9. Pedroso ERP, Oliveira RG. Blackbook: clínica médica. Belo Horizonte: Blackbook; 2007.

10. Duncan BB, Schmidt MI, Giugliani ERJ. Medicina ambulatorial: condutas de atenção primária baseadas em evidências. $3^{a}$ ed. Porto Alegre: Artmed; 2004.

11. Silva JSD, Almeida PHRF, Perini E, Pádua CAM, Rosa MB, Lemos GS. Erros de prescrição e administração envolvendo um medicamento potencialmente perigoso. Rev Enferm UFPE on line 2017;11(10):3707-17. https://doi.org/10.5205/reuol.1283430982-1-SM.1110201702

12. Brasil. Lei $n^{\circ} 5.991$, de 17 de dezembro de 1973. Dispõe sobre controle sanitário do comércio de drogas, medicamentos, insumos farmacêuticos e correlatos, e dá outras providências. Brasil; 1973.

13. Brasil. Lei $n^{\circ}$ 9.787, de 10 de fevereiro de 1999. Altera a Lei $n^{\circ} 6.360$, de 23 de setembro de 1976, que dispõe sobre a vigilância sanitária, estabelece o medicamento genérico, dispõe sobre a utilização de nomes genéricos em produtos farmacêuticos e dá outras providências. Brasil; 1999.

14. Brasil. Portaria $n^{\circ} 344$, de 12 de maio de 1998. Aprova o Regulamento Técnico sobre substâncias e medicamentos sujeitos a controle especial. Brasil; 1998.

15. Brasil. RDC $n^{\circ} 20$, de 05 de maio de 2011. Dispõe sobre o controle de medicamentos à base de substâncias classificadas como antimicrobianos, de uso sob prescrição, isoladas ou em associação. Brasil; 2011.

16. Madruga CMD, Souza ESM. Manual de orientações básicas para prescrição médica. Brasília: CRM-PB; 2011.

17. Instituto Brasileiro de Geografia e Estatística. Conheça cidades e estados do Brasil. Rio de Janeiro: Instituto Brasileiro de Geografia e Estatística. 2017 [acessado em 17 set. 2021]. Disponível em: https://cidades.ibge.gov.br

18. Prefeitura de Florianópolis. Relação Municipal de Medicamentos - REMUME. Lista de medicamentos disponibilizados pelo SUS em Florianópolis. 2017 [acessado em 5 janeiro 2022]. Disponível em: http://www.pmf.sc.gov.br/arquivos/arquivos/ pdf/13_10_2017_13.55.42.3cce57f5063705bcaa513f6ff952173c.pdf

19. Gomes LS, Oliveira ABM, Batista CL, Feitosa EL, Silva Júnior FTS, Mesquita MVN, et al. Avaliação da prescrição de medicamentos em uma unidade básica de saúde. In: I Congresso de Farmácia Clínica do Piauí - I CINFAPI. Teresina: Revista Interdisciplinar de Ciências Médicas; 2017.

20. Guzatto P, Bueno D. Análise de prescrições medicamentosas dispensadas na farmácia de uma unidade básica de saúde de Porto Alegre-RS. Rev HCPA \& Fac Med Univ Fed Rio Gd do Sul 2007;27(3):20-6. https://doi.org/10183/23585

21. Ferrari CKB, Brito LF, Oliveira CC, Moraes EV, Toledo OR, David FL. Falhas na prescrição e dispensação de medicamentos psicotrópicos: um problema de saúde pública. Rev Ciênc Farm Básica Apl 2013;34(1):109-16.

22. DATASUS. Sistema de informação de atenção básica: produção e marcadores. Rio Grande do Norte; 2015 [acessado em 17 set. 2021]. Disponível em: http://tabnet.datasus.gov.br

23. Jacobsen TF, Mussi MM, Silveira MPT. Análise de erros de prescrição em um hospital da região do sul do Brasil. Rev Bras Farm Hosp Saúde São Paulo 2015;6(3):23-6.

24. Ev LS, Guimarães AG, Castro VS. Avaliação das prescrições dispensadas em uma unidade básica de saúde do município de Ouro Preto, Minas Gerais, Brasil. Lat Am J Pharm 2008;27(4):543-7.

25. Laste C, Torres ILS, Deitos A, Souza AC, Souza A, Kauffmann C, et al. Análise de prescrições médicas dispensadas em farmácia no Sistema Único de Saúde. Rev HCPA 2013;33(1):15-25. https://doi.org/10183/157968

26. Silva LSN, Rocha MVIL, Batista AM. Prescrições de medicamentos sujeitos a controle especial em um município do Seridó Potiguar, Brasil. Infarma Cien Farm 2021;3(2):167-74. http://doi.org/10.14450/2318-9312.v33.e2.a2021.pp167-174

27. Silva Júnior FC; Batista AM. Prescrições de antimicrobianos em unidades básicas de saúde de um município do Seridó Potiguar, Brasil. Infarma Cien Farm 2019;31(4):271-6. http://doi.org/10.14450/2318-9312.v31.e4.a2019.pp271-276

28. Bandeira VAC, Hermann CTS, Siqueira CM, Oliveira KR. Análise das prescrições dispensadas em uma unidade básica de saúde do município de ljuí-RS. Saúde (Santa Maria) 2015;41(1):229-38. http://doi.org/10.5902/2236583415553

29. Alecrim JS, Castro JM, Miranda GM, Reinaldo FAF, Lacerda DCA, Reis JF, et al. Avaliação da prevalência de erros de prescrições recebidas em uma instituição de utilidade pública do Vale do Aço-MG. Ensaios e Ciência: Ciências Biológicas, Agrárias e da Saúde 2017;21(2):70-4. http://doi.org/10.17921/1415-6938.2017v21n2p70-74

30. World Health Organization. How to investigate drug use in health facilities: selected drug use indicators. Genebra: World Health Organization; 1993.

31. Organização Mundial da Saúde. Guia para a boa prescrição médica. Porto Alegre: Artmed; 1998.

32. Souza PHR, Oliveira MAS. Indicadores de prescrição médica: uma revisão sistemática 2015;27(1):5-13. http://doi. org/10.14450/2318-9312.v27.e1.a2015.pp5-13

33. Silva AS, Maciel GA, Wanderley LSL, Wanderley AG. Indicadores do uso de medicamentos na atenção primária de saúde: uma revisão sistemática. Rev Panam Salud Publica 2017;41:e132. http://doi.org/10.26633/RPSP.2017.132

34. Costa GMP, Oliveira MAS. Estudo das prescrições de psicotrópicos em uma farmácia da cidade de Sobral, Ceará, Brasil. Infarma Cien Farm 2017;29(1):27-33. http://doi.org/10.14450/2318-9312.v29.e1.a2017.pp27-33

35. Silva M, Oliveira APM, Pereira KD, Amaral KMM, Sousa MRN, Barros SS, et al. Avaliação do nível de conhecimento das prescrições em atenção primária à saúde. Research, Society and Development 2021;10(5):1-15. http://doi.org/10.33448/rsd-v10i5.14487

36. Zanetti MOB, Marchetti JM, Andrade RCG. Compreensão do usuário da atenção primária à saúde sobre o tratamento farmacológico: uma análise comparativa. J Appl Pharm Sci 2020;(7):1-10.

37. Torres MLD, Sousa LMG, Melo GC, Magalhães Júnior AAM, Firmo WCA. Prescrição de psicotrópicos e especialidade médica: estudo em uma farmácia comercial no município do Maranhão. Revista Científica do ITPAC 2014;7(4):1-6.

38. World Health Organization. Patient safety curriculum guide: multi-professional edition. Geneva: World Health Organization; 2011. 